Please do not remove this page

RMIT

UNIVERSITY

\title{
Surface acoustic wave solid-state rotational micromotor
}

Shilton, Richie; Langelier, Sean; Friend, James; Yeo, Leslie

https://researchrepository.rmit.edu.au/esploro/outputs/9921858611501341/filesAndLinks?institution=61RMIT_INST\&index=null

Shilton, R., Langelier, S., Friend, J., \& Yeo, L. (2012). Surface acoustic wave solid-state rotational micromotor. Applied Physics Letters, 100(3), 033503-1-033503-3. https://doi.org/10.1063/1.3676660 Document Version: Published Version

Published Version: https://doi.org/10.1063/1.3676660

Repository homepage: https://researchrepository.rmit.edu.au (C) 2012 American Institute of Physics

Downloaded On 2023/04/26 21:43:03 +1000

Please do not remove this page 


\title{
Surface acoustic wave solid-state rotational micromotor
}

\author{
Richie J. Shilton, ${ }^{a)}$ Sean M. Langelier, James R. Friend, and Leslie Y. Yeo ${ }^{\text {b) }}$ \\ Micro/Nanophysics Research Laboratory, RMIT University, Melbourne VIC 3000, Australia
}

(Received 16 November 2011; accepted 21 December 2011; published online 18 January 2012)

\begin{abstract}
Surface acoustic waves (SAWs) are used to drive a $1 \mathrm{~mm}$ diameter rotor at speeds exceeding $9000 \mathrm{rpm}$ and torque of nearly $5 \mathrm{nNm}$. Unlike recent high-speed SAW rotary motors, however, the present design does not require a fluid coupling layer but interestingly exploits adhesive stiction as an internal preload, a force usually undesirable at these scales; with additional preloads, smaller rotors can be propelled to $15000 \mathrm{rpm}$. This solid-state motor has no moving parts except for the rotor and is sufficiently simple to allow integration into miniaturized drive systems for potential use in microfluidic diagnostics, optical switching and microrobotics. (C) 2012 American Institute of Physics. [doi:10.1063/1.3676660]
\end{abstract}

There is increasing interest in the design of small-scale actuators, owing to the necessity for their use in many miniaturized systems such as portable microfluidic devices, microsurgical tools, insect-scale robots, micro-positioning stages, micro-optical systems, or as drive elements for a wide variety of applications. ${ }^{1-3}$ There are nevertheless considerable difficulties in driving rotation at these scales both with sufficient rotation speeds and torque, and without large and expensive power supplies, amplifiers, and oscillator circuits. In developing a simple method for driving truly portable small scale rotation, possibilities in scalable solid-state microactuators-previously complex at the microscale-become available.

Surface acoustic waves (SAWs) have been widely demonstrated for effectively driving both linear motion in microactuators ${ }^{4}$ and microfluidic rotation (among other things) ${ }^{5-7}$ for a wide range of functions. In the latter, fluid motion induced in liquid drops can be used as a coupling mechanism $^{8,9}$ to drive the spinning of a $5 \mathrm{~mm}$ mylar disc rotor at rotation speeds and radial accelerations exceeding $2250 \mathrm{rpm}$ and $172 \mathrm{~m} / \mathrm{s}^{2}$ for microcentrifugation. ${ }^{7}$ The necessity of a fluid coupling layer is nevertheless a severe limitation in applications where the presence of a fluid is outright inconvenient, such as in optical switches, integrated circuits, or sealed microactuators; further, the fluid could evaporate, be lost or misaligned, or become contaminated. Disc precession due to instabilities generated in the coupling fluid layer's meniscus also limits the maximum disc rotation speed to a fraction of what might be possible if the limit were due to the piezoelectric material instead, which is usually the case in actuators.

There has been limited success, however, in employing SAWs to drive rotation through dry friction akin to ultrasonic motors. ${ }^{3}$ A carousel SAW motor design has been proposed, employing steel ball bearings as a support and to transfer momentum from the substrate to the rotor to produce $\sim 180 \mathrm{rpm}$ rotation, without a stated torque and in a manner probably too complex and weak for most potential applications. ${ }^{10}$ Further, these complex designs suffer from limitations in scalability (e.g., the device was subsequently scaled down to

\footnotetext{
${ }^{a)}$ Present address: Micro/Nanophysics Research Laboratory, Monash University, Clayton VIC 3800, Australia.

b) Electronic mail: leslie.yeo@rmit.edu.au.
}

a $9 \mathrm{~mm}$ diameter rotor but achieved a maximum velocity of only $270 \mathrm{rpm}^{11}$ ), problems with wear, and the necessity for a significant preload. More recently, an arbitrary axis rotational SAW motor capable of driving $1 \mathrm{~mm}$ sphere rotors with a maximum velocity and torque of $1900 \mathrm{rpm}$ and $5.37 \mu \mathrm{Nm}$, respectively, has been developed. ${ }^{12}$ While the capability for multi-axis rotation lends itself to new applications, the design requires complex fabrication involving substrate drilling and the use of multi-axis interdigital transducers, thus limiting it to specific piezoelectric materials. Moreover, there is considerable difficulty in maintaining steady rotation, with velocities tapering off after $\sim 10 \mathrm{~ms}$.

We seek here to exploit dry friction to drive very high speed rotation of $1 \mathrm{~mm}$ diameter rotors using Rayleigh SAW with an exceptionally simple-and therefore miniaturizable_-design: a patterned piezoelectric chip, a miniaturized rotor, and an etched chamber to house the rotor. We show that the rotor geometry has substantial influence on the motor's performance and find that the rotor-substrate interaction is stiction-mediated in a way that may be useful for characterizing high-speed frictional micro/nanoscale phenomena. ${ }^{13-15}$ An electrode configuration is devised to impart rotation using focused transducers with integrated reflectors, borrowed from telecommunications applications, ${ }^{16}$ providing the ability to drive rotation at radial velocities over an order of magnitude faster that those previously described, with no moving parts other than the rotor itself.

Each rotor was housed in a chamber fabricated in Bosch deep reactive ion etched silicon $(\mathrm{Si})$, bonded with $\mathrm{UV}$ adhesive to a double-side polished, piranha-cleaned $127.68^{\circ} \mathrm{y}$-axis rotated, $\mathrm{x}$-axis propagating lithium niobate $(\mathrm{LN})$ wafer (University Wafer, South Boston, MA, USA) on which single phase unidirectional transducers (SPUDTs) ${ }^{17}$ operating at 29.7 MHz were fabricated (Fig. 1(a)). Specifically, a $175 \mathrm{~nm}$ gold layer was deposited on LN with a $5 \mathrm{~nm}$ chromium adhesion layer on which the SPUDTs were patterned using standard UV photolithography along with alignment marks for the Si chamber. Each SPUDT had 30 finger pairs, $3 \mathrm{~mm}$ front end aperture, and $\sim 16 \Omega$ impedance at resonance.

The $1 \mathrm{~mm}$ focusing SPUDTs were positioned along the $\mathrm{X}$-axis of the LN substrate, both facing towards the rotor chamber. To break the symmetry and hence induce rotation, each electrode was laterally offset from the center of the 

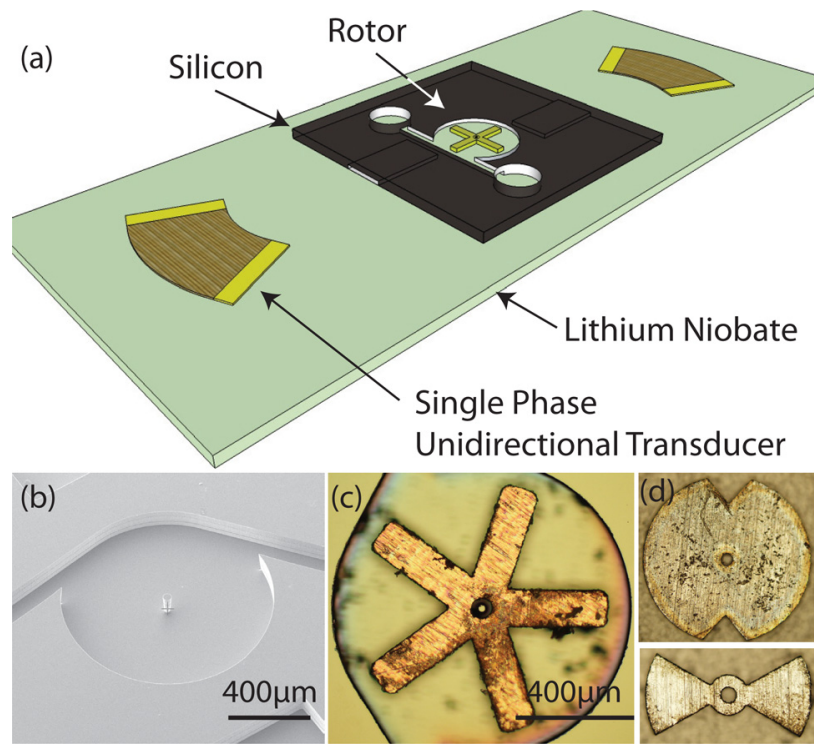

FIG. 1. (Color online) (a) Motor construction (not to scale); the entire device is around $32 \mathrm{~mm} \times 12 \mathrm{~mm} \times 1 \mathrm{~mm}$ and the top of the $\mathrm{Si}$ chamber is shown open for clarity. (b) Scanning electron microscope (SEM) image of the $\mathrm{Si}$ chamber used to house the rotors; this is viewed from the side that is bonded to the piezoelectric substrate; note the $40 \mu \mathrm{m}$ diameter Si central pin used to mount the rotors. (c) Miniaturized $(\sim 55 \mu \mathrm{m})$ thick steel rotor in an $80 \mu \mathrm{m}$ deep, $1 \mathrm{~mm}$ diameter Si chamber. (d) Disc shaped rotor with $85^{\circ}$, $160 \mu \mathrm{m}$ deep notches (top), and a $60^{\circ}$ angled "bow tie" rotor (bottom) (enhanced online) [URL: http://dx.doi.org/10.1063/1.3676660.1].

motor chamber by $0.5 \mathrm{~mm}$, as shown in Fig. 1(a). The rotors were kept aligned in the chamber with a $40 \mu \mathrm{m}$ diameter $\mathrm{Si}$ pin fabricated as part of the chamber (see Fig. 1(b)). Inlet and outlet ports to the chamber were drilled with a $1 \mathrm{~mm}$ diameter diamond drill bit in a drill press to allow nitrogen cleaning of the chamber. The structure was closed on the top with $\mathrm{Si}$; the schematic, however, shows an open structure to illustrate how the device operates. The rotors comprised $\sim 55 \mu \mathrm{m}$ thick sheets of mild steel with $1 \mathrm{~mm}$ nominal diameter. Motors were tested with circular "disc" shaped rotors and with 2-, 3-, $4-$, and 5-armed impeller rotors (a selection is shown in Figs. $1(\mathrm{c})$ and $1(\mathrm{~d}))$. Rotors were then placed in the Si chambers and the pieces were bonded to the LN chips with UV adhesive, with final glue thicknesses on the order of microns as measured using SEM (not shown). Due to the opaque $\mathrm{Si}$ chamber housing the rotor, the device was illuminated and viewed from underneath through the transparent $\mathrm{LN}$. To run the motor, an alternating current was applied at resonance to each SPUDT to generate a SAW with average surface velocities of $\sim 1 \mathrm{~ms}^{-1}$. The surface velocities were measured using a Laser Doppler Vibrometer (LDV, MSA-400, Polytec $\mathrm{GmbH}$, Waldbronn, Germany) across the center region where they contact the rotors. Rotor speeds were captured using high-speed video (Mikroton MC1310, Unterschleissheim, Germany), from which the rotational velocities were calculated using the software ImageJ (National Institutes of Health, Bethesda, MD, USA). We note that the motor behaviour was identical in both cases of the inlet and outlet ports being open or shut: while gas pumping may have been taking place, restricting the consequent "flow" had no effect on rotor behavior. The preload was estimated using a combination of precision scales and a vertical micropositioner by gradually releasing rotors from the substrate.

In Fig. 2(a), we observe the rotary speeds for a selection of rotors to exhibit first-order exponential asymptotic behaviour. Data regression with a least squares fit then allows an estimation of the maximum rotary speed and torque ${ }^{18}$; due to the first-order response, the actual speed and torque during use would be a linear interpolation between these maximum values, as shown in many other examples of such motors in the literature. ${ }^{19,20}$ The rotor speed does however oscillate about the first-order response, indicating rotor bouncing, which is very commonly observed in stick-slip piezoelectric motors, ${ }^{21}$ especially when loading is insufficient.

The rotors were found to rotate toward the source of acoustic radiation and in opposition to the propagation and energy flow direction of the SAW, at speeds between $10^{3}$ and $10^{4} \mathrm{rpm}$ (see Fig. 1 for video). This indicates that the rotor is in frictional contact with the substrate; the substrate's retrograde motion as the Rayleigh SAW propagates along would cause a surface in frictional contact with it to move in a direction opposing the SAW. Although another possible mechanism of rotor propulsion is acoustic levitation, ${ }^{22}$ this was not observed to be present in our system for two reasons: the direction and rotation speed of the rotor would have to be in the direction of the SAW propagation (away from the source of acoustic radiation) and beyond (up to four times the value of) $13400 \mathrm{rpm}$, respectively. ${ }^{22,23}$ The maximum rotor speed value is determined from the transverse vibration velocity used in the substrate (typically $1 \mathrm{~m} / \mathrm{s}$ ); for the Rayleigh wave in $\mathrm{LN}$, the in-plane vibration velocity is known to
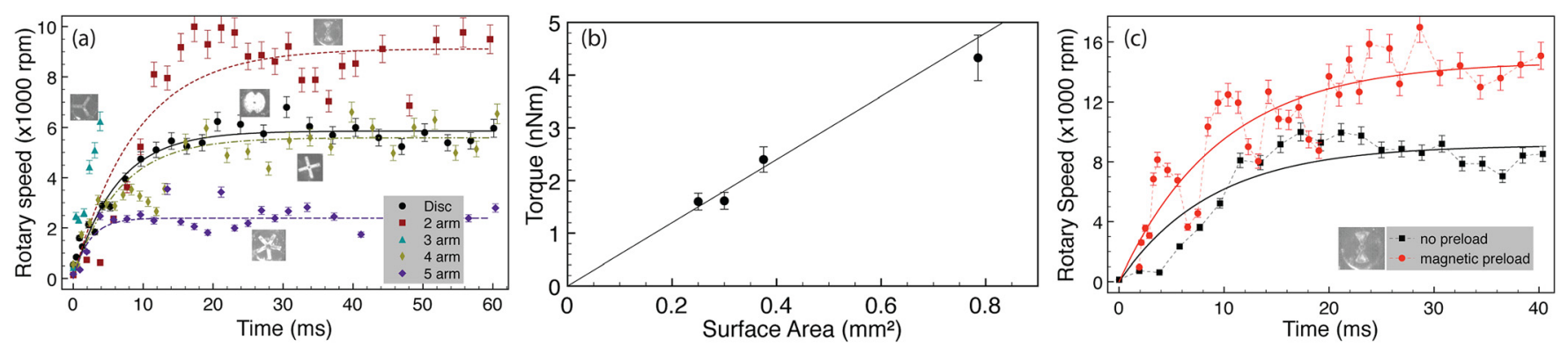

FIG. 2. (Color online) (a) Rotation speed for various rotor designs without applied preload. Due to its design asymmetry, we observed the rotor with 3 arms was displaced out-of-plane against the chamber roof, therefore impeding its rotation after an initial spin-up transient of around 5 ms. First-order exponential response least-squares fit to the data are shown for each rotor. (b) The start-up rotor torque reveals a linear relationship with respect to the surface area in contact with the SAW; the use of rotors with different geometries facilitated variations in the surface area. The linear fit shows a gradient of $c=5.7 \mathrm{nNm} / \mathrm{mm}^{2}$ for the steel rotors. (c) Comparison of the unloaded rotor speed with the preloaded case (approximately $220 \mu \mathrm{N} \pm 50 \mu \mathrm{N}$ ) shows a significant increase in steadystate velocities, at the cost of rotary speed stability. 
be 0.7 times this value at the substrate surface. ${ }^{24}$ Estimating the rotary speed to be this linear speed of $0.7 \mathrm{~m} / \mathrm{s}$ divided by the $0.5 \mathrm{~mm}$ rotor radius, we arrive at the stated approximate maximum rotor speed possible via friction.

This result is interesting since the rotors have no preload applied to enforce frictional contact. At these scales, adhesive stiction is of course present; ${ }^{25,26}$ usually undesirable, the surface energy reduction from the contact is sufficient to cause the rotor to adhere to the substrate. In fact, we find that the adhesive stiction force is almost an order of magnitude above the rotor weight force alone $(\sim 70 \mu \mathrm{N} \pm 10 \mu \mathrm{N})$, thus providing enough intrinsic preload to drive the rotors without the need for an external preload. From the Tabor and Bowden friction model, ${ }^{15}$ the maximum frictional force possible is directly proportional to the real contact area, which is typically far less than the apparent contact area. Since the surfaces are indeed adhering, the adhesion force is proportional to the apparent surface area projected by the rotor shape since the interacting surfaces, when brought close, have a reduction in surface energy proportional (not equal!) to the common area they share. Thus, the normal adhesion force is proportional to the apparent surface area, the friction force is proportional to the normal force multiplied by a complete friction slip coefficient $^{15}$ (at these speeds and considering the substrate vibration, the contact may be assumed to be completely slipping), and the torque is proportional to that friction force given the roughly similar rotor dimensions. This is why the start-up torque is proportional to the rotor surface area on the substrate, as seen in Fig. 2(b). This system may thus offer an interesting means to study friction at small scales under conditions of complete sliding to complete adhesion by reducing the substrate vibration amplitude. ${ }^{13-15}$ As the rotor torque is proportional to the frictional force, we calculate the friction slip coefficient $\mu_{\text {slip }} \approx T / r_{g} N$ to be $\sim 0.27 \pm 0.04$, where $T$ is the torque, $r_{g}$ the radius of gyration, and $N$ the normal force.

To investigate preload effects, we placed a ring magnet with an estimated preload of $\sim 220 \mu \mathrm{N} \pm 50 \mu \mathrm{N}$ under the chamber housing the fastest bow-tie rotor. Interestingly, this preload increased the rotation speed dramatically to over $14000 \mathrm{rpm}$ (Fig. 2(c)) but also increased rotor bouncing within the chamber-with a reduced friction slip coefficient $\sim 0.15 \pm 0.04$ (we note that this is not the typical static or dynamic friction coefficient). Further performance improve- ment should be possible by selecting materials and surface treatments that, ironically, have larger stiction or perhaps magnetic polarizability in order to increase the preload to a point where bouncing is suppressed. ${ }^{21}$

The authors are grateful for funding from a CSIRO Flagship Project Grant on Sensor Systems for Analysis of Aquatic Environments. Special thanks go to Ben Johnston (Laser Micromachining Solutions, OptoFab, Macquarie University) for his help with the rotor fabrication.

${ }^{1}$ Y. Suzuki, K. Tani, and T. Sakuhara, Sens. Actuators A: Phys. 83, 244 (2000).

${ }^{2}$ T. Morita, Sens. Actuators A: Phys. 103, 291 (2003).

${ }^{3}$ B. Watson, J. Friend, and L. Yeo, Sens. Actuators A: Phys. 152, 219 (2009).

${ }^{4}$ M. Kurosawa, M. Takahashi, and T. Higuchi, IEEE Trans. Ultrason. Ferroelectr. Freq. Control 43, 901 (1996).

${ }^{5} \mathrm{H}$. Li, J. Friend, and L. Yeo, Biomed. Microdev. 9, 647 (2007).

${ }^{6}$ J. R. Friend and L. Y. Yeo, Rev. Mod. Phys. 83, 647 (2011).

${ }^{7}$ R. Shilton, N. Glass, P. Chan, L. Yeo, and J. Friend, Appl. Phys. Lett. 98, 254103 (2011).

${ }^{8}$ R. Hodgson, M. Tan, L. Yeo, and J. Friend, Appl. Phys. Lett. 94, 024102 (2009).

${ }^{9}$ Y. Bourquin, J. Reboud, R. Wilson, and J. Cooper, Lab Chip 10, 1898 (2010).

${ }^{10}$ G. Zhang, L. Cheng, S. Zhang, J. Yu, and X. Shui, Electron. Lett. 36, 1437 (2000).

${ }^{11}$ L. Cheng, G. Zhang, S. Zhang, J. Yu, and X. Shui, Ultrasonics 39, 591 (2002).

${ }^{12}$ R. Tjeung, M. Hughes, L. Yeo, and J. Friend, Appl. Phys. Lett. 99, 214101 (2011).

${ }^{13}$ R. Capozza, A. Vanossi, A. Vezzani, and S. Zapperi, Phys. Rev. Lett. 103, 085502 (2009).

${ }^{14}$ M. Urbakh, J. Klafter, D. Gourdon, and J. Israelachvili, Nature 430, 525 (2004).

${ }^{15}$ O. M. Braun, Tribol. Lett. 39, 283 (2010).

${ }^{16}$ D. Royer and E. Dieulesaint, Elastic Waves in Solids: Free and Guided Propagation (Springer, Berlin, 2000), Vol. 1.

${ }^{17} \mathrm{C}$. Hartmann and B. Abbott, in Proceedings of the IEEE Ultrasonics Symposium (IEEE, Piscataway, NJ, 1989), pp. 79-89.

${ }^{18}$ K. Nakamura, M. Kurosawa, H. Kurebayashi, and S. Ueha, IEEE Trans. Ultrason. Ferroelectr. Freq. Control 38, 481 (1991).

${ }^{19}$ J. Friend, K. Nakamura, and S. Ueha, IEEE Trans. Ultrason. Ferroelectr. Freq. Control 52, 1343 (2005).

${ }^{20}$ J. Friend, L. Yeo, and M. Hogg, Appl. Phys. Lett. 92, 014107 (2008).

${ }^{21}$ K. Liu, J. Friend, and L. Yeo, Phys. Rev. E 80, 046201 (2009).

${ }^{22}$ Y. Hashimoto, Y. Koike, and S. Ueha, J. Acoust. Soc. Am. 103, 26 (1998).

${ }^{23}$ T. Ide, J. Friend, K. Nakamura, and S. Ueha, Sens. Actuators A: Phys. 135, 740 (2007).

${ }^{24}$ C. Tseng, J. Appl. Phys. 38, 4281 (1967).

${ }^{25}$ W. M. van Spengen, R. Puers, and I. D. Wolf, J. Micromech. Microeng. 12, 702 (2002).

${ }^{26}$ M. Müser and M. Urbakh, Adv. Chem. Phys. 126, 187 (2003). 